\title{
Implications of MODIS bow-tie distortion on aerosol optical depth retrievals, and techniques for mitigation
}

\author{
A. M. Sayer ${ }^{1,2}$, N. C. Hsu ${ }^{1}$, and C. Bettenhausen ${ }^{1,3}$ \\ ${ }^{1}$ NASA Goddard Space Flight Center, Greenbelt, Maryland, USA \\ ${ }^{2}$ Goddard Earth Sciences Technology And Research (GESTAR), Universities Space \\ Research Association (USRA), Columbia, Maryland, USA \\ ${ }^{3}$ Science Systems and Applications Inc., Lanham, Maryland, USA \\ Correspondence to: A. M. Sayer (andrew.sayer@nasa.gov)
}

Received: 20 July 2015 - Published in Atmos. Meas. Tech. Discuss.: 18 August 2015

Revised: 4 December 2015 - Accepted: 7 December 2015 - Published: 17 December 2015

\begin{abstract}
The scan geometry of the Moderate Resolution Imaging Spectroradiometer (MODIS) sensors, combined with the Earth's curvature, results in a pixel shape distortion known as the "bow-tie effect". Specifically, sensor pixels near the edge of the swath are elongated along-track and across-track compared to pixels near the centre of the swath, resulting in an increase of pixel area by up to a factor of $\sim 9$ and, additionally, the overlap of pixels acquired from consecutive scans. The Deep Blue and Dark Target aerosol optical depth (AOD) retrieval algorithms aggregate sensor pixels and provide level 2 (L2) AOD at a nominal horizontal pixel size of $10 \mathrm{~km}$, but the bow-tie distortion means that they also suffer from this size increase and overlap. This means that the spatial characteristics of the data vary as a function of satellite viewing zenith angle (VZA) and, for $\mathrm{VZA}>30^{\circ}$, corresponding to approximately $50 \%$ of the data, are areally enlarged by a factor of $50 \%$ or more compared to this nominal pixel area and are not spatially independent of each other. This has implications for retrieval uncertainty and aggregated statistics, causing a narrowing of AOD distributions near the edge of the swath, as well as for data comparability from the application of similar algorithms to sensors without this level of bow-tie distortion. Additionally, the pixel overlap is not obvious to users of the L2 aerosol products because only pixel centres, not boundaries, are provided within the L2 products. A two-step procedure is proposed to mitigate the effects of this distortion on the MODIS aerosol products. The first (simple) step involves changing the order in which pixels are aggregated in L2 processing to reflect geographical location rather than scan order, which removes the bulk of the
\end{abstract}

overlap between L2 pixels and slows the rate of growth of L2 pixel size vs. VZA. This can be achieved without significant changes to existing MODIS processing algorithms. The second step involves additionally changing the number of sensor pixels aggregated across-track as a function of VZA, which preserves L2 pixel size at around $10 \mathrm{~km} \times 10 \mathrm{~km}$ across the whole swath but would require algorithmic quality assurance tests to be re-evaluated. Both of these steps also improve the extent to which the pixel locations a user would infer from the L2 data products represent the actual spatial extent of the L2 pixels.

\section{Introduction}

The Moderate Resolution Imaging Spectroradiometers (MODIS) aboard the Terra and Aqua platforms have been used to create data products for a range of earth science disciplines, including analyses of the atmospheric aerosol burden. Mid-visible aerosol optical depth (AOD) data products have been generated routinely by a variety of dedicated algorithms over bright (Hsu et al., 2004, 2006, 2013) and dark (Kaufman et al., 1997; Levy et al., 2007, 2013; Hsu et al., 2013) land surfaces, ocean surfaces (Tanré et al., 1997; Levy et al., 2013), and as a by-product of algorithms for the atmospheric correction of land/ocean surface reflectance (e.g. Ahmad et al., 2010; Lyapustin et al., 2011).

The level 2 (L2; orbit-level) MODIS dedicated aerosol data product is known as MOD04 for data generated from MODIS Terra and MYD04 for data from MODIS 
Aqua (hereafter MXD04 collectively). The latest version of MXD04 products (Collection 6, C6) contains data generated by the Deep Blue (DB) algorithms over land surfaces (Hsu et al., 2013), a Dark Target (DT) over-land algorithm, and an over-water algorithm which also uses wavelengths at which the water surface is dark (Levy et al., 2013). These L2 products are generated from level $1 \mathrm{~b}(\mathrm{~L} 1 \mathrm{~b})$ calibrated radiance data measured by the MODIS instruments. To decrease noise in the retrieval and provide a manageable data volume, the standard L2 products are provided at a nominal horizontal pixel size of $10 \mathrm{~km}$ (referred to as "retrieval pixels") compared to a L1b pixel size of $0.25-1 \mathrm{~km}$ (dependent on band, referred to as "sensor pixels"). As aerosol horizontal variations are often on length scales of the order of tens of kilometres (e.g. Anderson et al., 2003), this pixel size should preserve the underlying spatial shape of aerosol fields without major smoothing of features. Note that a separate nominal $3 \mathrm{~km}$ DT product is also available, albeit with larger uncertainties than the $10 \mathrm{~km}$ standard product (Munchak et al., 2013; Remer et al., 2013, Livingston et al., 2014).

However, a consequence of the MODIS scan geometry and Earth's curvature, of which many users of MXD04 data may not be aware, is a distortion of pixel size and shape from the centre to the edge of the swath. This is known as the "bow-tie effect" and has the potential to alias into angledependent artefacts and changes in retrieval quality in the derived data sets. It also presents a challenge for the continuation of MODIS-like data sets using similar sensors such as the Visible Infrared Imaging Radiometer Suite (VIIRS) on the Suomi National Polar-orbiting Partnership (S-NPP) platform, launched in late 2011, for which these bow-tie distortions are much smaller. Consequently, data sets generated from similar algorithms may exhibit different angulardependent characteristics.

The purpose of this study is to illustrate the influence of the MODIS bow-tie distortion on AOD retrievals and examine some techniques for mitigation of these distortions, for potential application in future MODIS (or VIIRS) data reprocessings. Section 2 discusses MODIS and its scan geometry, providing an illustration of the bow-tie effect and the dependence of MODIS AOD retrievals on view angle. Section 3 proposes a two-step solution to mitigate these effects, of which the first step is relatively simple and could be accomplished with minor changes to existing MODIS processing algorithms, while the second step would require more careful evaluation. Finally, Sect. 4 provides a discussion on the importance of the results.

\section{Illustration of the problem}

MODIS takes measurements in a total of 36 bands with central wavelengths between $412 \mathrm{~nm}$ and $14.4 \mu \mathrm{m}$ (Barnes et al., 1998; Toller et al., 2013). The L1b data are organised into 5 min granules, which consist of 1354 pixels across-track and 203 scans along-track. The term "along-track" indicates the

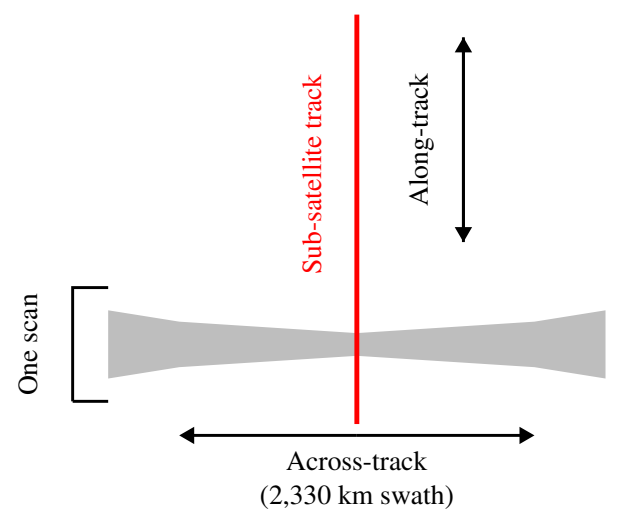

Figure 1. Illustration of some terms related to MODIS scan geometry.

direction of the satellite flight track (for daytime data this is approximately from north to south for Terra and from south to north for Aqua, due to the satellites' $\sim 98.2^{\circ}$ inclination), while "across-track" indicates the direction perpendicular to the flight track (alternating east-west and west-east for successive scans). This is illustrated in Fig. 1.

Each scan is approximately $10 \mathrm{~km}$ wide at nadir; there are 10 detectors for nominal $1 \mathrm{~km}$ bands, 20 detectors for $0.5 \mathrm{~km}$ bands, and 40 detectors for the two bands at nominal $0.25 \mathrm{~km}$ resolution. Note that these quoted nominal pixel sizes are only approximate, and this terminology is used for simplicity of understanding. Barnes et al. (1998) and Xiong et al. (2006) provide more details about MODIS spatial characterisation. The MODIS spatial response functions on a pixel level are not exactly square and have some slight blurring between adjacent pixels. For example, pre-launch characterisation of MODIS Terra revealed band 8 (centred near $412 \mathrm{~nm}$ ), nominally of $1 \mathrm{~km}$ pixel size, had an effective across-track size around $1.08 \mathrm{~km}$ across-track and $1.01 \mathrm{~km}$ along-track. Distortions are band dependent and, for the bands relevant to AOD retrieval, of similar (fairly small) magnitude. In the present study pixel sizes (lengths) are approximated as half the distance between adjacent pixel centres in each direction. The error introduced by this approximation is much smaller than the distortion of pixel shape/size resulting from the bowtie effect. Still it is worth bearing in mind that the representation of sensor pixels (and coarser-resolution L2 data) as quadrilaterals is an idealisation. Campagnolo and Montano (2014) provide some more discussion and on-orbit characterisation of MODIS's spatial performance from the point of view of land surface reflectance data products.

In the L1b products, the data are available both at native resolution and aggregated to the footprints of the coarser bands. For example, MODIS bands 1 and 2, centred near 650 and $860 \mathrm{~nm}$ respectively, are recorded with a nominal $0.25 \mathrm{~km}$ pixel size; however, they are additionally provided aggregated to the footprints of the $0.5 \mathrm{~km}$ bands (which is native resolution for bands 3-7) and $1 \mathrm{~km}$ bands (which is 
(a) MODIS Terra, 10:20 UTC, 08 Aug 2012

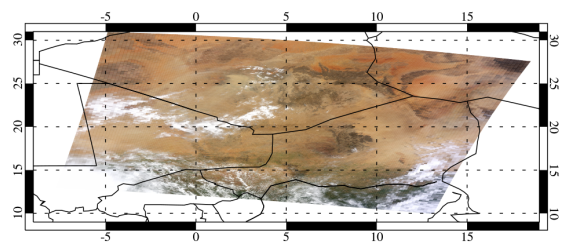

(c) Deep Blue AOD at $550 \mathrm{~nm}$

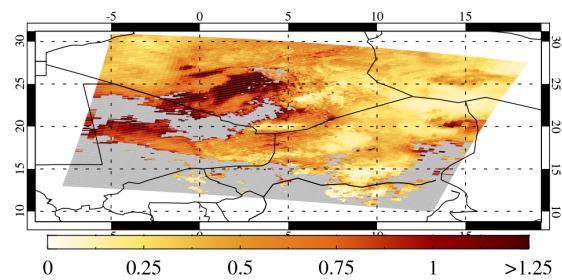

(b) Sensor (viewing) zenith angle, degrees

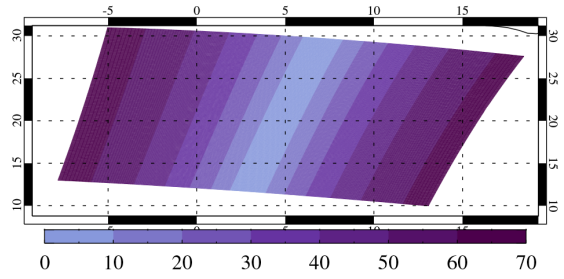

(d) Retrieval pixel area, $\mathrm{km}^{2}$

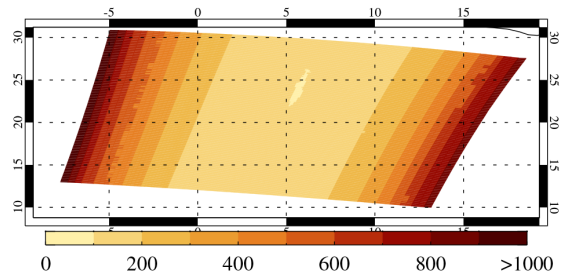

Figure 2. MODIS granule used as a case study throughout this manuscript. Panel (a) shows a true-colour composite, (b) the VZA along and across the scans, (c) the L2 (nominal $10 \mathrm{~km}$ ) Deep Blue retrieved AOD for the granule, and (d) the Deep Blue retrieval-pixel area.

(a) MODIS pixel growth vs. view angle

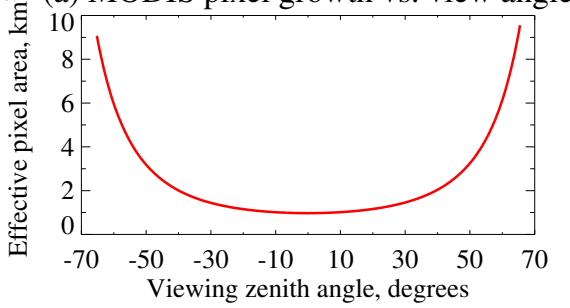

(b) View zenith angle histogram

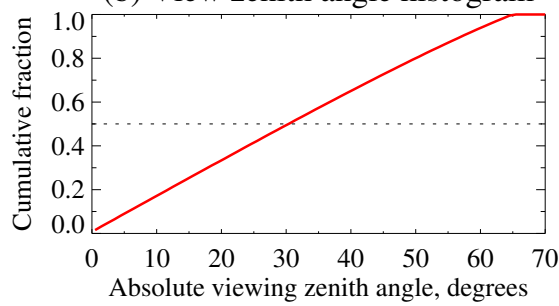

Figure 3. (a) Area of nominal $1 \mathrm{~km} \times 1 \mathrm{~km}$ sensor pixels as a function of VZA, and (b) histogram of absolute VZA. The median is shown with a dashed line. Data for the MODIS granule shown in Fig. 2.

native resolution for bands 8-36). DB uses the L1b data aggregated to $1 \mathrm{~km}$ while the DT algorithms use the L1b data at $0.5 \mathrm{~km}$.

The L2 aerosol products are created by aggregating blocks of contiguous sensor pixels through one scan along-track and $101 \mathrm{~km}$ sensor pixels across-track (or $200.5 \mathrm{~km}$ pixels for the appropriate bands in DT). The DB algorithms (Hsu et al., 2013) retrieve AOD from suitable (e.g. cloud-free snow-free) data at sensor-pixel resolution and then aggregate to retrieval-pixel resolution (a "retrieve-then-average" technique), while the DT algorithms (Levy et al., 2013) average measured reflectance from suitable pixels and then perform a single retrieval at retrieval-pixel resolution (an "averagethen-retrieve" technique). The two averaging methods should be equivalent when the underlying scene is homogeneous but not when there is surface or atmospheric heterogeneity. Thus, the L2 aerosol products consist of 135 retrievalpixel positions across-track and 203 along-track (the four excess across-track nominal $1 \mathrm{~km}$ sensor pixels are discarded as 1354 does not divide evenly by 10). As the sensor scans across-track back and forth, the light observed by MODIS is reflected from a scan mirror onto the focal plane assemblies.
Dependent on the scan direction, both sides of this scan mirror are used. Differences in the quality of the characterisation of these two mirror sides can lead to striping in the data between forward and reverse scans in some situations (Franz et al., 2007).

Because of this scan geometry the spatial resolution degrades from nadir (viewing zenith angle (VZA) $0^{\circ}$ ) to the scan edge $\left(\mathrm{VZA} \sim 65^{\circ}\right.$ ), such that pixels near the edge of the scan are larger than those near nadir; the swath width is $\sim 2330 \mathrm{~km}$, despite the fact that there are only 1354 acrosstrack sensor pixels. The primary distortion is across-track (i.e. pixels get longer in the longitudinal direction) but there is also an along-track (i.e. latitudinal) distortion, resulting in overlap between pixels from consecutive scans near the swath edges. These give a scan a shape similar to a bow tie, hence the term "bow-tie effect".

These effects are illustrated for an example MODIS Terra granule in Figs. 2 and 3. This granule is the basis for most of the results shown in this study. Note that slight asymmetries and discontinuities in the retrieval-pixel size result because of variations in terrain elevation across the granule. Although the nominal $10 \mathrm{~km} \times 10 \mathrm{~km}$ horizontal resolution 

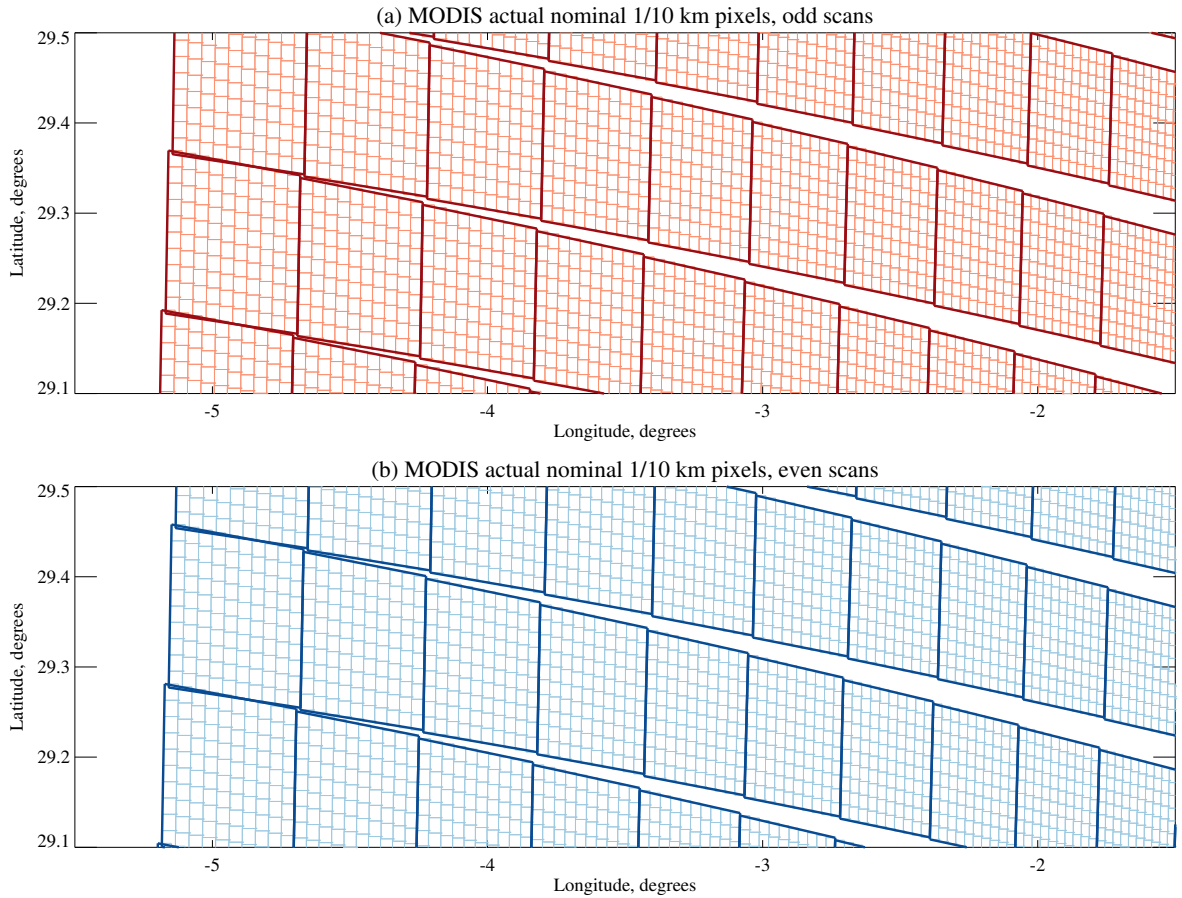

(c) MODIS actual nominal $1 / 10 \mathrm{~km}$ pixels, all scans

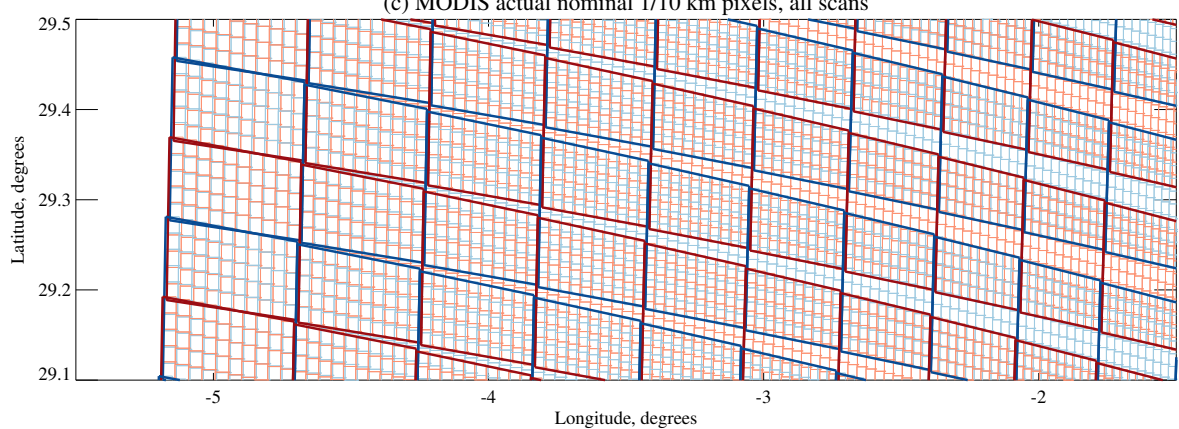

Figure 4. Borders of nominal $1 \mathrm{~km}$ sensor pixels (pale colours) and nominal $10 \mathrm{~km}$ retrieval pixels (strong colours) along the western edge of the granule shown in Fig. 2, illustrating the overlap between scans near swath edges. Data from alternating forward/backward scans are shown in red and blue. Panel (a) shows odd-numbered scans only, (b) even-numbered scans only, and (c) all scans.

results in a retrieval-pixel area of $\sim 100 \mathrm{~km}^{2}$ near the centre of the swath, as the VZA increases, the pixel area is increased by almost a factor of 10 at the extreme edges of the swath. Figure 3 shows that the median absolute VZA is about $30^{\circ}$, for which pixel area is increased by around $\sim 50 \%$ compared with VZA $=0^{\circ}$. Hence, although the MODIS aerosol retrieval-pixel size is commonly stated to be $10 \mathrm{~km} \times 10 \mathrm{~km}$, half the time the actual area encompassed by these retrieval pixels is at least $50 \%$ larger than that. Note that the sign of VZA in Fig. 3 is defined such that positive values are found on the western side of the swath.

Taking a closer view of the area near the western edge of the swath, Fig. 4 shows the ground locations of the sensor and retrieval pixels. Scans are coloured alternating in red and blue (odd scans in red, even in blue). Two factors are immediately apparent. One is the strong elongation of pixels in the across-track (longitudinal) direction. The second is that, at the swath edge, successive scans (e.g. a red-coloured scan and the blue-coloured scans immediately before and after it) are fully overlapped in the along-track direction. Each edgeof-swath retrieval covers half of the area of the retrieval pixel from the scan before it and half of the area from the scan after it (i.e. they are fully overlapping). Even 10 retrievals in from the scan edge the retrieval pixels are more than half overlapped between successive scans. This therefore represents a sizeable fraction of the MXD04 data, particularly in terms of area covered.

This overlap is not obvious to L2 data product users, as the L2 files only present the central latitudes and longitudes for the retrieval pixels. Figure 5 shows the same retrievalpixel locations as in Fig. 4, except with the retrieval-pixel boundaries which would be inferred from the L2 central 


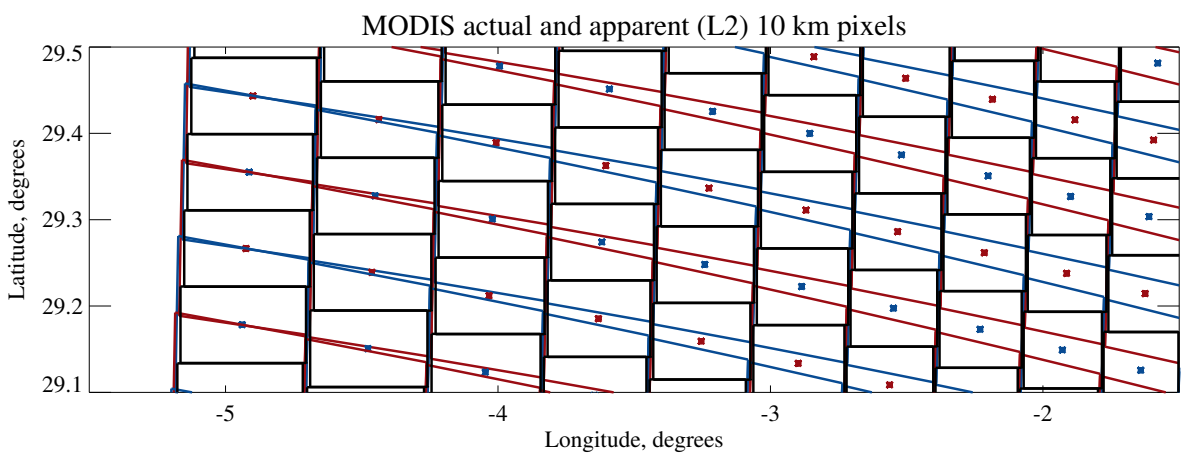

Figure 5. Borders of nominal $10 \mathrm{~km}$ retrieval pixels (strong colours, as in Fig. 4c) along the western edge of the granule shown in Fig. 2. Pixel centres are overplotted with coloured asterisks, and inferred L2 pixel bounds are drawn in black lines.

(a) Deep Blue arid

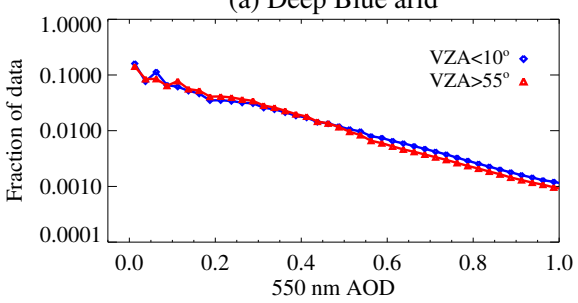

(c) Dark Target land

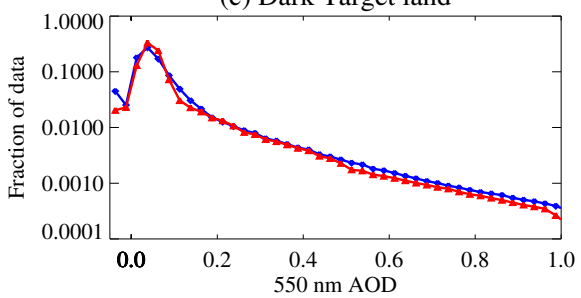

(b) Deep Blue vegetated

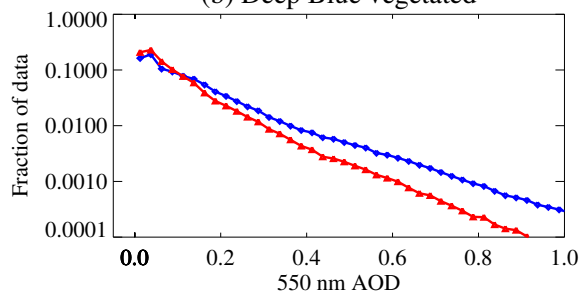

(d) Dark Target ocean

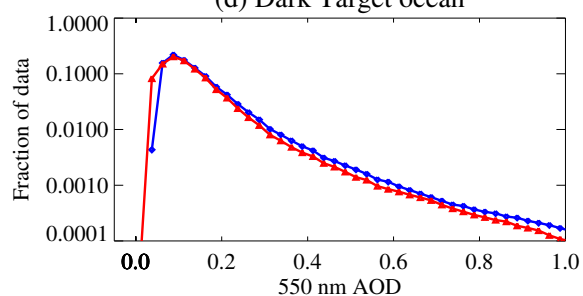

Figure 6. Histograms of retrieved MODIS Aqua AOD at $550 \mathrm{~nm}$ during the year 2006 retrieved from (a) DB over bright arid surfaces, (b) DB over dark vegetated surfaces, (c) DT over dark vegetated surfaces, and (d) the over-water DT algorithm.

latitudes/longitudes overplotted. The fact that the L2 pixels overlap and are oversampled is not clear from the L2 products alone and would require auxiliary knowledge of the L1b sensor-pixel locations, which are not provided in the L2 products.

All other things being equal, the expected effects of both aspects of this distortion (i.e. pixel enlargement and overlap) on the MXD04 products would be to decrease the variability of the retrieved AOD near the edge of the scan compared to that near the centre of the scan. Figure 6 shows histograms of AOD at $550 \mathrm{~nm}$ from all four algorithm/surface types included within the C6 MXD04 products (DB over arid and vegetated surfaces; DT over land and ocean), for data with $\mathrm{VZA}<10^{\circ}$ and $\mathrm{VZA}>55^{\circ}$, corresponding to roughly the lowest and highest VZA quintiles respectively (Fig. 3). These histograms are calculated from all L2 products for the year 2006; for the DT ocean panel only latitudes poleward of $35^{\circ}$ are used, as otherwise a sampling bias may be introduced due to the presence of sun glint near the centre of the swath in tropical regions.

The median, standard deviations, and width of the central $68 \%$ of the data (i.e. difference between 84th and 16th percentiles of AOD) for these histograms are shown in Table 1. In all cases, the retrievals near the edge of the swath show smaller variability than those near the centre, which is consistent with the expected effects of the bow-tie distortion. The median AOD is more stable between the two sets of VZA. The decrease in variability $(\sim 25 \%$ decrease in AOD standard deviation or width of central $68 \%$ ) is most pronounced for the dark (vegetated) land surfaces in DB and DT. This may reflect the lack of strong aerosol point sources over the open ocean and potentially the spatial scales of aerosol features over desert and ocean surfaces being larger than aerosols over vegetated land.

Note that the differences between DB and DT histogram shapes over dark vegetated surfaces are due in part to algorithmic assumptions; for example, what surface counts as 
Table 1. Median, standard deviation, and width of central $68 \%$ of AOD at $550 \mathrm{~nm}$ for the histograms shown in Fig. 6.

\begin{tabular}{lllll}
\hline Algorithm & $\begin{array}{l}\text { Abs. VZA } \\
\text { range }\end{array}$ & $\begin{array}{l}\text { Median } \\
\text { AOD }\end{array}$ & $\begin{array}{l}\text { Standard } \\
\text { deviation of AOD }\end{array}$ & $\begin{array}{l}\text { Width of } \\
\text { central 68 \% }\end{array}$ \\
\hline DB arid & $<10^{\circ}$ & 0.14 & 0.30 & 0.38 \\
DB vegetated & $>55^{\circ}$ & 0.15 & 0.29 & 0.35 \\
& $>10^{\circ}$ & 0.09 & 0.15 & 0.21 \\
DT vegetated & $<15^{\circ}$ & 0.06 & 0.11 & 0.14 \\
& $>55^{\circ}$ & 0.05 & 0.18 & 0.13 \\
DT ocean & $<10^{\circ}$ & 0.05 & 0.15 & 0.09 \\
& $>55^{\circ}$ & 0.11 & 0.20 & 0.13 \\
& & & & 0.13 \\
\hline
\end{tabular}

dark vs. bright in the two algorithms, and the fact that DT over land allows retrievals of small negative AOD (down to -0.05 ) while DB does not. All histogram shapes may also be influenced by the fact that the algorithms' AOD retrieval uncertainties are factors of solar/viewing geometry (e.g. Hyer et al., 2011; Sayer et al., 2013, 2014); depending on the nature of these uncertainties, they may also be contributing to shifts in the shapes of the AOD distributions. It is difficult to disentangle the relative importance of these oversampling and error reduction to the change in width, particularly since the answers are likely to be algorithm specific. However, Table 1 and Fig. 6 support the assertion that the retrieval overlap and size distortion, undesirable from a point of view of homogeneity of data characteristics, may also be influencing the statistics of the retrieved AOD.

\section{Potential mitigation techniques}

\subsection{Reordering along-track sensor-pixel aggregation}

The most straightforward way to ameliorate the effects of the bow-tie distortion on the MXD04 aerosol products would simply be to change the along-track sensor-pixel aggregation order from one based on the order of data collection (i.e. scan order) to one based on the order in which pixel centres are arranged on the ground (i.e. geographical order). This could be achieved by reordering the L1b data at each across-track position within each granule in order of increasing (for Aqua) or decreasing (for Terra) latitude, and it would not require any other changes to data processing algorithms or output data file format. Algorithmically, it is as simple as looping across each of the across-track positions and sorting the data by latitude (although for granules straddling the poles it becomes a little more complicated). This is referred to hereafter as the "resorted" aggregation technique and has the basic effect of decreasing the distortion in the along-track direction.

A comparison between swath-edge L2 standard and resorted retrieval-pixel boundaries is shown in Fig. 7. The $100 \%$ areal overlap of swath-edge pixels in the standard aggregation is reduced to around $40 \%$ in the resorted aggregation and effectively removed by a distance of a halfdozen retrieval pixels in to the swath (while in the stan- dard aggregation pixels are still significantly overlapped at this position). Additionally, although the spatial overlap is $\sim 40 \%$, the overlap in terms of L1b pixel radiance data is only $\sim 20 \%$, as the central (non-overlapped) $60 \%$ of the resorted retrieval pixel contains data from 8 out of the 10 alongtrack positions within the cell. This resorting technique also means that the actual area covered by each pixel now aligns much more closely with the area that a data user would infer based on the geolocation information within the L2 data products (cf. Fig. 5).

Because of the DB retrieve-then-average methodology, it is most straightforward to use the DB algorithm to illustrate the effects of changing the aggregation technique on AOD retrievals than DT. Figure 8 shows the difference in AOD and pixel area for this technique as compared to the standard L2 aggregation (Fig. 2). The large-scale AOD patterns do not change, although some striping is reduced (as pixels from scans using both mirror sides are now included in the same retrieval pixel, mitigating residual calibration effects on AOD discontinuities). The median AOD difference is, as expected, negligible $(<0.01)$ and the standard deviation of the AOD difference is 0.02 . The main advantage from this approach, however, is the reduction of retrieval-pixel area by up to around $40 \%$ (Fig. 8d) at the edge of scan compared to the standard aggregation, due to the removal of much of the along-track retrieval-pixel growth and overlap. For VZA $>30^{\circ}$, the increase of pixel area with VZA proceeds only about half as quickly for a factor of around 5 at the edge of the scan compared to around a factor of 9 using the standard aggregation.

\subsection{Change of across-track pixel aggregation counts}

Although the resorting technique described in Sect. 3.1 increases retrieval-pixel independence and decreases pixel size by addressing the along-track aspect of the bow-tie distortion, it does not improve the across-track pixel growth vs. VZA, which is the larger of the two contributions to retrievalpixel size increase. In an ideal situation retrieval-pixel size and shape would not vary with across-track scan position, but for MODIS this can only be accomplished by changing the number of pixels aggregated across-track when going from sensor-pixel to L2 retrieval-pixel resolution.

This has larger potential implications for the MXD04 product than only performing the resorting aggregation, because quality assurance (QA) procedures used to identify L2 retrievals suitable for quantitative analysis (Hsu et al., 2013; Levy et al., 2013) depend on the statistics of the data within the L2 retrieval pixel, including the number of available sensor pixels for retrieval, and additionally because the size of the resulting L2 data arrays would change, which may affect some data users. Thus it is likely that changing the acrosstrack pixel aggregation (in addition to applying the resorting technique of Sect. 3.1 to mitigate along-track distortion) would change the error characteristics of the MXD04 prod- 


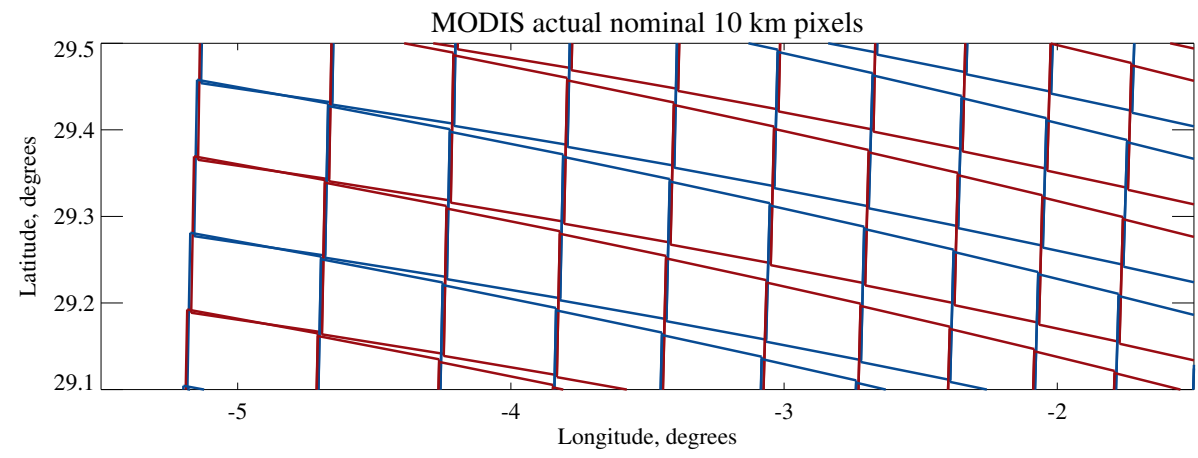

(b) MODIS resorted nominal $10 \mathrm{~km}$ pixels

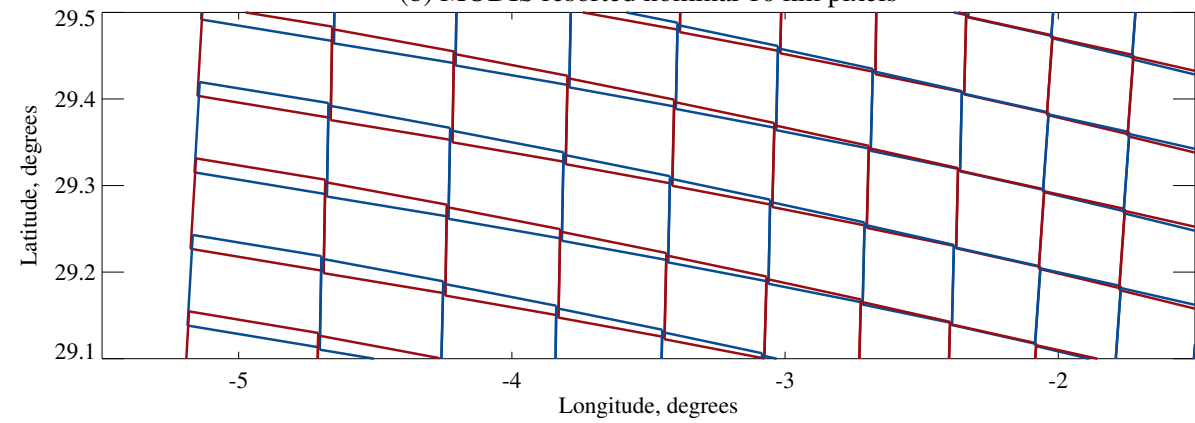

Figure 7. Actual L2 retrieval-pixel boundaries for (a) the standard (Figs. 4c and 5) and (b) resorted aggregation techniques.

(a) DB AOD, resorted aggregation

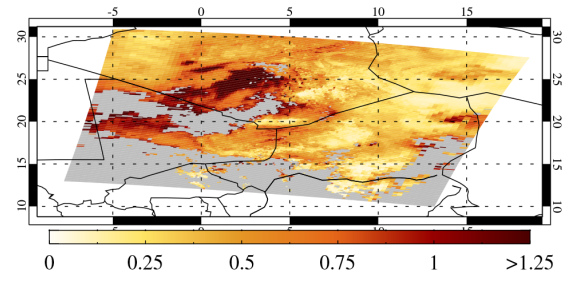

(c) MODIS retrieval pixel area, resorted, $\mathrm{km}^{2}$

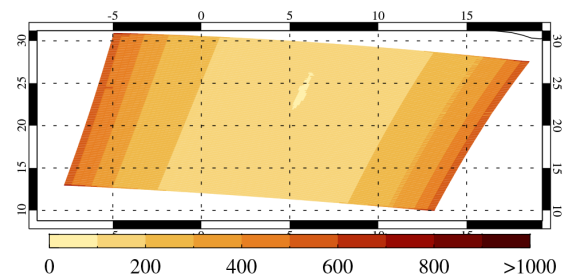

(b) Reaggregation AOD difference

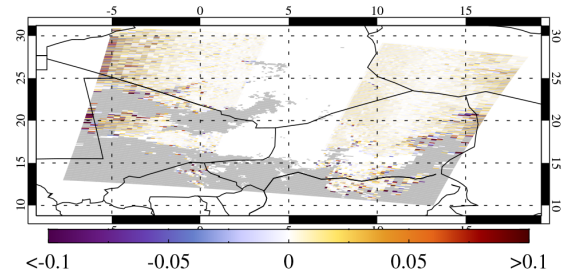

(d) MODIS area ratio (resorted/original)

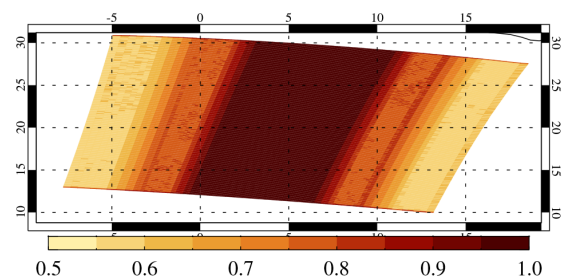

Figure 8. DB AOD retrieval for the granule in Fig. 2 using the "resorted" aggregation technique. Panel (a) shows the DB AOD at 550 nm, (b) the difference from the AOD using the standard aggregation technique (Fig. 2c), (c) the resulting retrieval-pixel area, and (d) the ratio of the area using the resorted technique to that from the standard technique (i.e. ratio of panel (c) to Fig. 2d). Pixels without retrievals are shaded in grey.

ucts more significantly and require more careful assessment prior to large-scale implementation.

The combination of resorting along-track and changing across-track aggregation number is referred to hereafter as the "variable aggregation" technique. Figure 9 shows that by changing the number of across-track positions aggregated as a function of VZA, from 2 near the edge of the swath to 10 (as in the standard MXD04 aggregation) near the centre, it is possible to preserve a pixel area of around $100 \mathrm{~km}^{2}$ across the whole MODIS swath. This results in an increase of L2 data array size from $203 \times 135$ retrieval pixels to $203 \times 233$ retrieval pixels. Note that 10 pixels are still aggregated alongtrack, so near the edge of the swath a total of 20 L1b sensor pixels would contribute to the L2 retrieval pixel, while 
(a) Across-track pixel aggregation

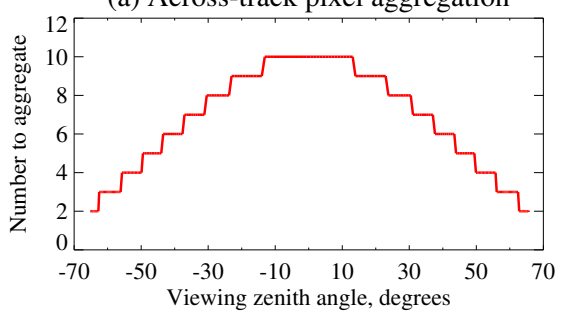

(b) L2 pixel area, $\mathrm{km}^{2}$

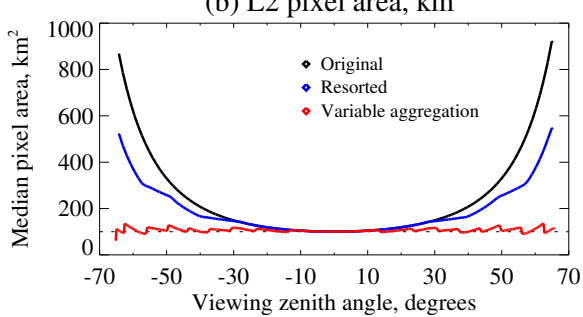

Figure 9. (a) Number of across-track positions aggregated for the "variable aggregation" technique, and (b) pixel area as a function of VZA for the granule shown in Fig. 2 for all three aggregation techniques. The dashed line indicates an area of $100 \mathrm{~km}^{2}$.

(a) MODIS Terra, 10:20 UTC, 08 Aug 2012

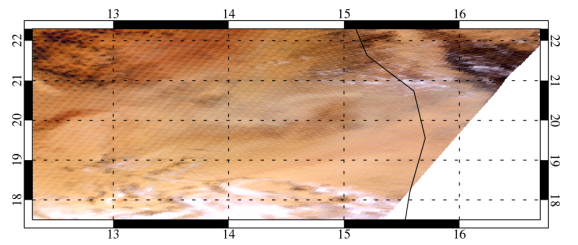

(c) AOD, resorted aggregation

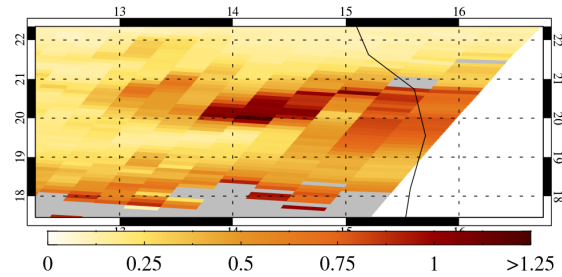

(b) AOD, standard aggregation

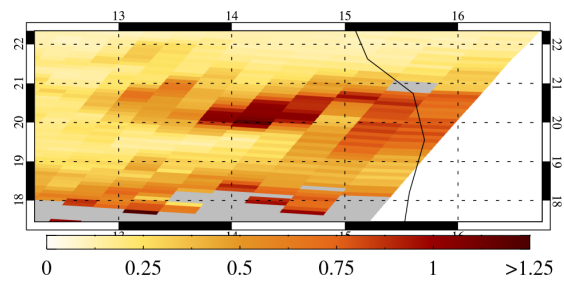

(d) AOD, variable aggregation

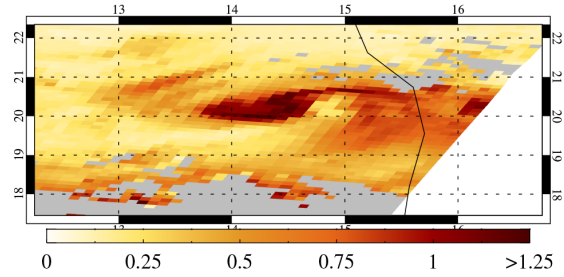

Figure 10. Effect of aggregation technique on spatial distribution of retrieved DB AOD at $550 \mathrm{~nm}$. Panel (a) shows a true-colour image for the eastern portion of the granule shown in Fig. 2, and panels (b-d) show the L2 AOD for each of the standard, resorted, and variable aggregation techniques respectively. Pixels without retrievals are shaded in grey.

for VZA smaller than $\sim 10^{\circ}$ the aggregation remains the same as in the standard product (i.e. 100 sensor pixels per L2 pixel). One further point of relevance, however, is that since the source L1b sensor pixels are still distorted and the atmospheric path length is also dependent on VZA, it is still possible that the error characteristics of the retrievals, and efficacy of cloud tests making use of spatial homogeneity (e.g. Martins et al., 2002), could change as a function of VZA.

One potential downside of moving to the "variable aggregation" technique is the decrease in the number of L1b pixels available to contribute to the L2 pixel near the edge of the swath. One of the original reasons for reporting the L2 data at a coarser spatial resolution than the L1b products was to decrease the level of noise in the AOD retrieval, and so a drop in the number of pixels averaged could potentially increase the noise. Larger-scale testing would be required to assess this in detail. However, it is not expected to be a significant factor: for DB, in most cases the standard deviation of the sensor-pixel AOD retrievals within the L2 grid is small (often 0.01 or less), suggesting little noise or instability within the retrieval. This is consistent with validation analyses which indicate that the bulk of the retrieval error in a given situation is contextual (i.e. dependent on geometry, surface cover, and aerosol loading/type) rather than radiometric random noise (Sayer et al., 2013, 2014). Additionally, the geometries which would be subject to reaggregation are also those geometries where retrieval errors tend to be lower due to an increased atmospheric path length, which also has the effect of suppressing noise in the retrieval, and would still be true after applying the reaggregation.

Because of the preservation of horizontal pixel size vs. VZA, the variable aggregation technique has larger implications for the spatial distribution of retrieved AOD than the resorted technique (Sect. 3.1). Figure 10 shows part of the eastern edge of the granule from Fig. 2, comparing the spatial distributions of AOD for each aggregation method. Note that the retrieval pixels are drawn here corresponding to their locations which would be inferred from the L2 geolocation information. The standard and resorted methods show similar spatial features; the decrease in striping from the resorted aggregation is obvious. The variable aggregation technique reveals the structure of the visible dust plumes with much 
better fidelity. Data holes are also much more closely aligned with the presence of clouds; while this will not affect the L2 retrievals themselves (as only cloud-free sensor pixels are used), it does improve the utility of the data for those wishing to assess the dependence of aerosol properties as a function of distance from clouds (e.g. Bar-Or et al., 2011; Várnai et al., 2013) or collocate aerosol and cloud data for other purposes.

\subsection{Larger-scale test application}

As a larger-scale test of the effect of reaggregation on AOD statistics, the proposed techniques have been applied to MODIS Terra DB data from the year 2006 over part of eastern North America $\left(30-50^{\circ} \mathrm{N}, 70-90^{\circ} \mathrm{W} ; 1978\right.$ granules total), which uses almost exclusively the "vegetated" branch of the DB processing code. Although these results are specific to this region and may well differ for regimes dominated by other aerosol types, or for different algorithms, they do provide an indication of the magnitude of changes in AOD which may result from reaggregation of the MODIS data.

The standard QA tests required for inclusion in the DB "best estimate" data product (i.e. pixels with $\mathrm{QA}=2$ or 3 ) were applied (Hsu et al., 2013), with the exception that the absolute pixel count criterion of at least 40 (out of 100) valid retrievals within the $10 \times 10$ sensor-pixel box being required was altered to a relative criterion of at least $40 \%$ of potential pixels being present. Without this change, the variable aggregation technique would lose coverage near the edge of the swath, since these reaggregated retrieval pixels have fewer potential sensor-pixel retrievals to draw from for large VZA (Fig. 9). Due to the improved homogeneity of retrieval-pixel size with scan angle in the variable aggregation technique, this run provides a factor of 1.79 more L2 retrievals than either the standard or resorted aggregation methods (which have a very similar data volume to each other). This is higher than that expected from the ratio of potential L2 pixels within a granule using the different techniques $(233 / 135 \approx 1.73)$; the extra may be a result of more accurately collocating adjacent sensor pixels in areas close to data gaps (such as coasts or cloud edges).

Figure 11 shows the distribution of AOD as a function of VZA for all three aggregation techniques. The distributions for the original and resorted techniques are barely distinguishable. However, the distribution for the variable aggregation technique clearly shows an increased width at large VZA compared to the other two (by 0.01-0.02). The increase comes mainly from the higher-AOD tail of the distribution and also slightly (of order 0.01 or less) increases the median AOD at large VZA compared to the other two techniques. This is consistent with expectations, as otherwise the bowtie effect would act to smooth out high-AOD scenes to a greater extent than low-AOD scenes (since high-AOD scenes are more likely to be heterogeneous).

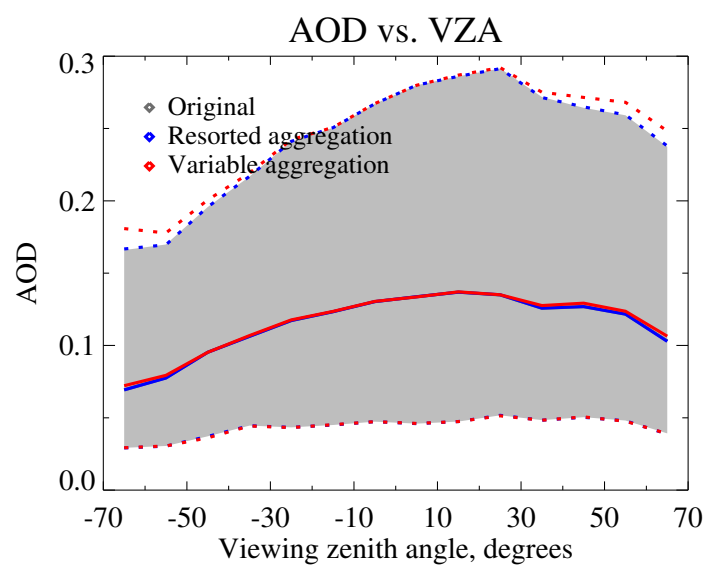

Figure 11. Effect of aggregation technique on angular distribution of AOD over eastern North America in 2006, in bins of $10^{\circ}$. Solid lines show the median AOD in each bin, and dashed lines bound the central $68 \%$ of the data. The shaded grey region indicates the central $68 \%$ of data using the operational MODIS C6 aggregation technique.

The distributions of AOD vs. VZA do still show some residual angular dependence of peak and width for all aggregation techniques. This is likely due to a combination of effects from sampling and residual angular-dependent uncertainties in the DB AOD retrieval of these surfaces. Comparing the widths of the AOD distributions near the edges and centre of the swath in Fig. 11 suggests that, for this region, around a third to a half of the difference in AOD distribution width results from the artificial smoothing from the bow-tie distortion, while the remainder may be a combination of effects from changing retrieval uncertainty characteristics and sampling differences. Since the change in AOD distributions is most pronounced on the high-AOD tail, however, it is likely that regions with a higher typical level of AOD, or more frequent high-AOD events, would exhibit larger changes in AOD distributions as a result of aggregation changes.

Spatially, the patterns of AOD are, as expected, very similar. Figure 12 shows the mean and standard deviation of AOD for the standard and variable aggregation runs at $0.5^{\circ}$ resolution across the whole year (L3-like data, computed from daily averages). Note that geometric, rather than arithmetic, mean/standard deviation are shown, as AOD distributions are close to lognormal rather than normal (e.g. O'Neill et al., 2000). The variable aggregation technique results in a slightly higher AOD (in most cases by less than 0.01); the effect on AOD temporal standard deviation is more mixed. This is consistent with Figure 11, since the minimisation of the bow-tie distortion with the variable aggregation technique acts to reduce artificial smoothing of the AOD field, increasing the AOD slightly at large VZA. The mixed sign of the effect on temporal standard deviation results because noise in AOD temporal variability is decreased (by decreas- 

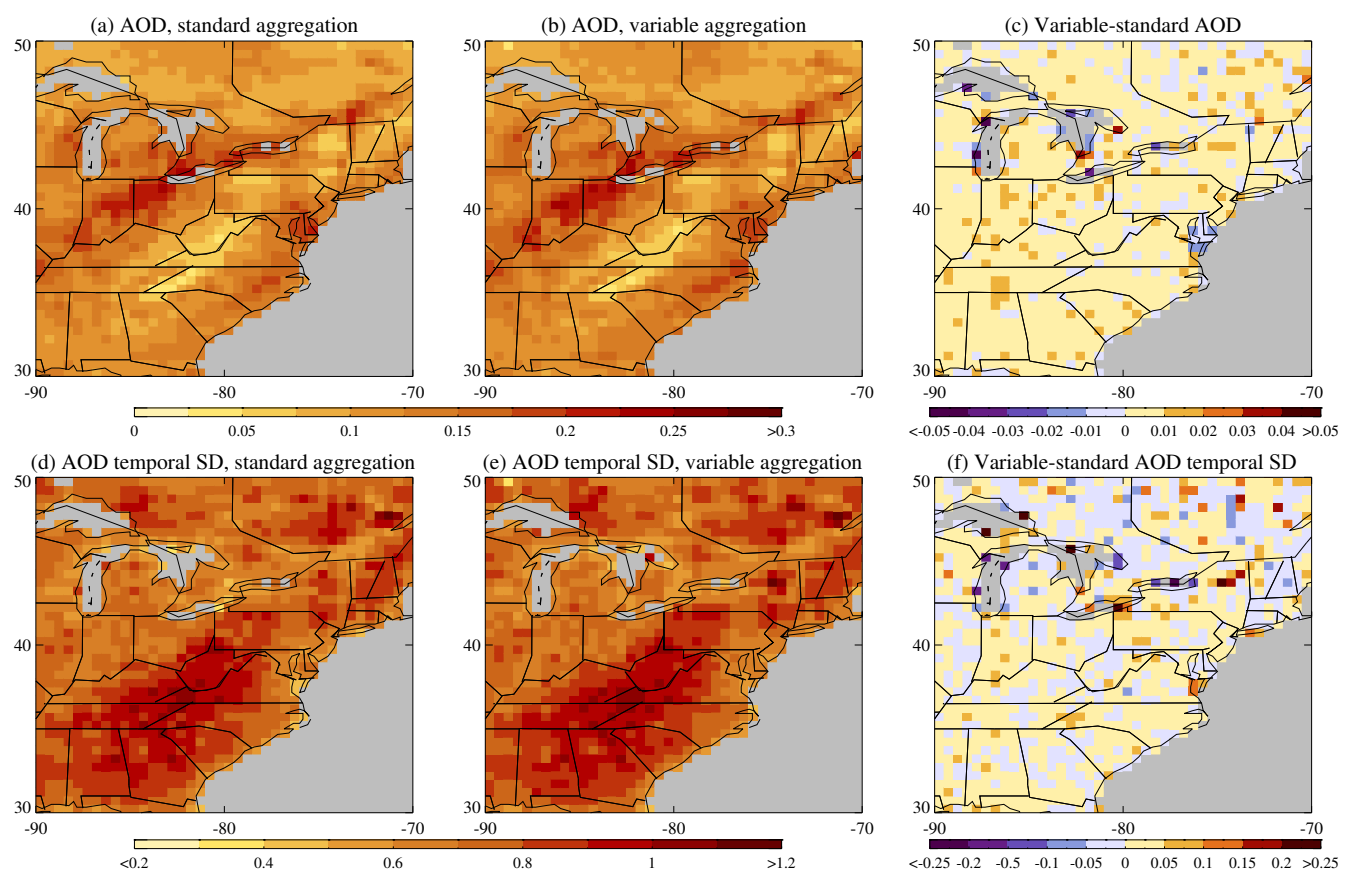

Figure 12. Effect of aggregation technique on spatial distribution of AOD over eastern North America in 2006 vs. VZA. The top and bottom rows show geometric mean and temporal standard deviation respectively. The left column shows data for the standard aggregation, the middle the variable aggregation technique, and the right the difference between the two. Data shown at $0.5^{\circ}$ resolution; grid cells with fewer than 10 days of data are shaded in grey.

ing the VZA dependence of AOD, given data from a single day typically samples only a small range of VZA) but the slight increase in AOD can also mean an increase in AOD standard deviation. Again, it is important to emphasise that differences will be region specific since they depend on the magnitude and spatiotemporal variability of AOD.

\section{Perspectives on product regularity and MODIS/VIIRS continuity}

The full influence of the MODIS bow-tie effect on the MXD04 aerosol data products has not received wide recognition up to this point. Despite the fact that visualisations of the data make the elongation of L2 pixels fairly obvious, the substantial overlap between them at the edge of the swath, and the effect that this oversampling and enlargement may have on the L2 data and aggregated statistics, has been underappreciated, and many data users may be unaware of it because it cannot be inferred from the L2 data products in isolation.

Although this distortion has not hindered the widespread use of the MXD04 data products for scientific applications, the issue is increasingly relevant now that MODIS-like algorithms are being developed for application to NPP-VIIRS data. The VIIRS sensor makes measurements at similar spectral bands to MODIS, with a similar L1b sensor-pixel size of nominal $0.75 \mathrm{~km}$ for moderate-resolution bands (M-bands), and a broader swath $(3040 \mathrm{~km})$. VIIRS has a similar across- track scanning pattern to MODIS, although with $16 \mathrm{M}$-band detectors per scan. However, VIIRS incorporates several design features to reduce the bow-tie distortion (Wolfe et al., 2012, 2013). Firstly, the VIIRS on-board native pixel size is actually smaller than the nominal M-band size in the acrosstrack direction. The scan is divided into three regions (in both directions). From nadir out to a scan angle of $31.72^{\circ}$, three pixels are aggregated across-track to create the M-band L1b data; from 31.72 to $44.86^{\circ}$ two pixels are aggregated, and from $44.86^{\circ}$ to the edge of scan no aggregation is performed. Additionally, at the outer two aggregation zones, two and four pixels respectively are deleted from the along-track scan edges (so-called "bow-tie deletion"). The net effect of this is that pixels within each aggregation zone are enlarged in length by at most approximately only a factor of 2 and that overlap between sensor pixels from consecutive scans is in most cases two or fewer detectors, compared to up to $100 \%$ in MODIS. Thus, there are structural reasons that, even if the AOD retrieval algorithms are as close as possible, the characteristics of L2 and L3 data derived from the sensors may differ, which has implications for MODIS/VIIRS data continuity and the generation of multi-sensor long-term climate data records.

It is therefore recommended that future reprocessings of the MODIS aerosol data records consider ways to mitigate the effect of the bow-tie distortion on the spatial structure of the AOD products. In the meantime this study is intended 
to serve as a point of reference to bring these issues to wider recognition within the data user community. Aggregating sensor pixels by geographical location rather than in along-track scan order is one straightforward step which can be taken to decrease the along-track distortion; however, as discussed, taking the additional step of changing to a variable across-track aggregation has larger implications for algorithm development and QA tests. This is already an issue which algorithm developers are facing to an extent, because with the VIIRS bow-tie deletion the number of valid sensor pixels also changes as a function of VZA through the three M-band aggregation zones.

One important caveat to this recommendation is that a strength of the MODIS data product suite is that multiple L2 products are produced on a common grid (e.g. standard nominal $10 \mathrm{~km} \mathrm{DB}$ and DT aerosol data) or on a common scaling of this detector-based grid (e.g. some cloud products). This makes it fairly straightforward to, for example, perform direct comparisons between the DB and DT aerosol data sets or to collocate aerosol and cloud data products for other studies. If one product were reaggregated then it would make the most sense for others to also be reaggregated to preserve this common structure. Note, however, that many other MODIS data products are essentially ungridded aside from the underlying scan structure (e.g. some nominal $1 \mathrm{~km}$ cloud or ocean colour products) or on a grid defined relative to the Earth rather than the satellite (e.g. some land products), in which case comparisons or synergistic applications would be unaffected by the change to atmospheric product aggregation (since the grids are not the same as the aerosol products anyway). In fact, the L2 grids of the MODIS land products were designed in part to account for some of the scan-related distortions (Wolfe et al., 1998).

The present study has focused on mid-visible AOD, as this is the primary data product from the DB/DT L2 aerosol algorithms. Other retrieved quantities, such as AOD at other wavelengths or the Ångström exponent, are susceptible to these effects in the same way. The effect on downstream L3 products such as mean AOD in a $1^{\circ}$ box is likely to be smaller on global average since these products represent an additional level of spatial aggregation anyway. However the increase in effective spatial resolution near the edge of the swath resulting from the variable aggregation technique would lead to a greater number of available L2 retrievals to go into the L3 average, potentially resulting in a more accurate grid-box mean AOD. Additionally, the L3 products contain histograms of retrieved AOD; by decreasing effective pixel size and removing the overlap between pixels, these histograms should also become more accurate representations of the aerosol variability within a grid cell. Indeed, a test application to data over North America reveals changes in L3-like data due to changes in off-nadir retrievals. Changes are, however, likely to be algorithm- and regionspecific. Therefore, it seems likely that the benefits of reag- gregation would extend to the $\mathrm{L} 3$ aerosol data products, even though the most substantial benefit is to the L2 products.

Although presented here in the context of the DB and DT AOD retrieval algorithms, these types of issues will be common to any algorithm which aggregates MODIS data to a coarser resolution or to any other sensor with a similar type of scanning geometry and detector setup. The mitigation is fairly straightforward for this example because the MXD04 spatial resolution matches the along-track length of one scan (i.e. 10 nominal $1 \mathrm{~km}$ sensor pixels). However for data products where the L2 resolution does not match the scan length, such as the DT nominal $3 \mathrm{~km}$ AOD product (Remer et al., 2013), the distortion will have an irregular impact on the actual and perceived size of the L2 retrieval pixels. Thus, to obtain a L2 product where the pixel-to-pixel variation in area covered is minimised, it makes most sense to perform retrievals at either the coarsest L1b data pixel size (i.e. nominal $1 \mathrm{~km}$ for MODIS or $0.75 \mathrm{~km}$ for VIIRS) or else with an along-track aggregation which is an exact divisor (or multiple) of the scan length (e.g. 2, 5, or $10 \mathrm{~km}$ for MODIS; 3, 6, or $12 \mathrm{~km}$ for VIIRS).

Acknowledgements. This work was supported by the NASA EOS program, managed by $\mathrm{H}$. Maring. More information about Deep Blue can be found at http://deepblue.gsfc.nasa.gov. The MODIS Characterization Support Team and Ocean Biology Processing Group are thanked for their extensive efforts in maintaining the high radiometric quality of MODIS data. MODIS level 1 data and aerosol products are available from http://ladsweb.nascom.nasa.gov. The authors thank three referees (L. A. Munchak and two anonymous) for their helpful reviews of this manuscript.

Edited by: A. Kokhanovsky

\section{References}

Ahmad, Z., Franz, B. A., McClain, C. R., Kwiatowska, E. J., Werdell, J., Shettle, E. P., and Holben, B. N.: New aerosol models for the retrieval of aerosol optical thickness and normalized water-leaving radiances from the SeaWiFS and MODIS sensors over coastal regions and open oceans, Appl. Optics, 49, 55455560, doi:10.1364/AO.49.005545, 2010

Anderson, T. L., Charlson, R. J., Winker, D. M., Ogren, J. A., and Holmén, K.: Mesoscale Variations of Tropospheric Aerosols, J. Atmos. Sci., 60, 119-136, doi:10.1175/15200469(2003)060<0119:MVOTA>2.0.CO;2, 2003.

Bar-Or, R. Z., Altaratz, O., and Koren, I.: Global analysis of cloud field coverage and radiative properties, using morphological methods and MODIS observations, Atmos. Chem. Phys., 11, 191-200, doi:10.5194/acp-11-191-2011, 2011.

Barnes, W. L., Pagano, T. S., and Salomonson, V. V.: Prelaunch characteristics of the Moderate Resolution Imaging Spectroradiometer (MODIS) on EOS-AM1, IEEE T. Geosci. Remote, 36, 1088-1100, doi:10.1109/36.700993, 1998. 
Campagnolo, M. L., and Montano, E. L.: Estimation of Effective Resolution for Daily MODIS Gridded Surface Reflectance Products, IEEE T. Geosci. Remote, 52, 5622-5632, doi:10.1109/TGRS.2013.2291496, 2014.

Franz, B. A., Kwiatkowska, E. J., Meister, G., and McClain, C. R.: Utility of MODIS-Terra for ocean color applications, P. SPIE IS\&T Elect. Im., 6677, doi:10.1117/12.732082, 2007.

Hsu, N. C., Tsay, S.-C., King, M. D., and Herman, J. R.: Aerosol properties over bright-reflecting source regions, IEEE T. Geosci. Remote, 42, 557-569, doi:10.1109/TGRS.2004.824067, 2004.

Hsu, N. C., Tsay, S.-C., King, M. D., and Herman, J. R.: Deep Blue retrievals of Asian aerosol properties during ACE-Asia, IEEE T. Geosci. Remote, 44, 3180-3195, doi:10.1109/TGRS.2006.879540, 2006.

Hsu, N. C., Jeong, M.-J., Bettenhausen, C., Sayer, A. M., Hansell, R., Seftor, C. S., Huang, J., and Tsay, S.-C.: Enhanced Deep Blue aerosol retrieval algorithm: the second generation, J. Geophys. Res., 118, 9296-9315, doi:10.1002/jgrd.50712, 2013.

Hyer, E. J., Reid, J. S., and Zhang, J.: An over-land aerosol optical depth data set for data assimilation by filtering, correction, and aggregation of MODIS Collection 5 optical depth retrievals, Atmos. Meas. Tech., 4, 379-408, doi:10.5194/amt-4-379-2011, 2011.

Kaufman, Y. J., Wald, A. E., Remer, L. A., Gao, B.-C., Li, R.-R., and Flynn, L.: The MODIS $2.1 \mu \mathrm{m}$ channel-correlation with visible reflectance for use in remote sensing of aerosol, IEEE T. Geosci. Remote, 35, 1286-1298, doi:10.1109/36.628795, 1997.

Levy, R. C., Remer, L. A., Mattoo, S., Vermote, E. F., and Kaufman, Y. J.: Second-generation operational algorithm: retrieval of aerosol properties over land from inversion of Moderate Resolution Imaging Spectroradiometer spectral reflectance, J. Geophys. Res., 112, D13211, doi:10.1029/2006JD007811, 2007.

Levy, R. C., Mattoo, S., Munchak, L. A., Remer, L. A., Sayer, A. M., Patadia, F., and Hsu, N. C.: The Collection 6 MODIS aerosol products over land and ocean, Atmos. Meas. Tech., 6, 29893034, doi:10.5194/amt-6-2989-2013, 2013.

Livingston, J. M., Redemann, J., Shinozuka, Y., Johnson, R., Russell, P. B., Zhang, Q., Mattoo, S., Remer, L., Levy, R., Munchak, L., and Ramachandran, S.: Comparison of MODIS $3 \mathrm{~km}$ and $10 \mathrm{~km}$ resolution aerosol optical depth retrievals over land with airborne sunphotometer measurements during ARCTAS summer 2008, Atmos. Chem. Phys., 14, 2015-2038, doi:10.5194/acp-142015-2014, 2014.

Lyapustin, A., Wang, Y., Laszlo, I., Kahn, R., Korkin, S., Remer, L., Levy, R., and Reid, J. S.: Multiangle implementation of atmospheric correction (MAIAC): 2. Aerosol algorithm, J. Geophys. Res., 116, D03211, doi:10.1029/2010JD014986, 2011.

Martins, J. V., Tanré, D., Remer, L., Kaufman, Y., Mattoo, S., and Levy, R.: MODIS Cloud screening for remote sensing of aerosols over oceans using spatial variability, Geophys. Res. Lett., 29, 1619, doi:10.1029/2001GL013252, 2002.
Munchak, L. A., Levy, R. C., Mattoo, S., Remer, L. A., Holben, B. N., Schafer, J. S., Hostetler, C. A., and Ferrare, R. A.: MODIS 3 $\mathrm{km}$ aerosol product: applications over land in an urban/suburban region, Atmos. Meas. Tech., 6, 1747-1759, doi:10.5194/amt-61747-2013, 2013.

O’Neill., N. T., Ignatov, A., Holben, B. N., and Eck, T. F.: The lognormal distribution as a reference for reporting aerosol optical depth statistics; Empirical tests using multi-year, multi-site AERONET Sunphotometer data, Geophys. Res. Lett., 27 (20), 3333-3336, doi:10.1029/2000GL011581, 2010.

Remer, L. A., Mattoo, S., Levy, R. C., and Munchak, L. A.: MODIS $3 \mathrm{~km}$ aerosol product: algorithm and global perspective, Atmos. Meas. Tech., 6, 1829-1844, doi:10.5194/amt-6-1829-2013, 2013.

Sayer, A. M., Hsu, N. C., Bettenhausen, C., and Jeong, M.-J.: Validation and uncertainty estimates for MODIS Collection 6 "Deep Blue" aerosol data, J. Geophys. Res., 118, 7864-7872, doi:10.1002/jgrd.50600, 2013.

Sayer, A. M., Munchak, L. A., Hsu, N. C., Levy, R. C., Bettenhausen, C., and Jeong, M.-J.: MODIS Collection 6 aerosol products: comparison between Aqua's e-Deep Blue, Dark Target, and "merged" data sets, and usage recommendations, J. Geophys. Res., 119, 13965-13989, doi:10.1002/2014JD022453, 2014.

Tanré, D., Kaufman, Y. J., Herman, M., and Mattoo, S.: Remote sensing of aerosol properties over oceans using the MODIS/EOS spectral radiances, J. Geophys. Res., 102, 1697116988, doi:10.1029/96JD03437, 1997.

Toller, G., Xiong, X., Sun, J., Wenny, B. N., Geng, X., Kuyper, J., Angal, A., Chen, H., Madhavan, S., and Wu, A.: Terra and Aqua moderate-resolution imaging spectroradiometer collection 6 level 1B algorithm, J. Appl. Remote Sens., 7, 073557, doi:10.1117/1.JRS.7.073557, 2013.

Várnai, T., Marshak, A., and Yang, W.: Multi-satellite aerosol observations in the vicinity of clouds, Atmos. Chem. Phys., 13, 38993908, doi:10.5194/acp-13-3899-2013, 2013.

Wolfe, R. E., Roy, D., P., and Vermote, E.: MODIS Land Data Storage, Gridding, and Compositing Methodology: Level 2 Grid, IEEE Trans. Geosci. Remote Sens., 36, doi:10.1109/36.701082, 1998.

Wolfe, R. E., Lin, G., Nishihama, M., Tewari, K. P., and Montano, E.: NPP VIIRS early on-orbit geometric performance, P. SPIE IS\&T Elect. Im., 8510, doi:10.1117/12.929925, 2012.

Wolfe, R. E., Lin, G., Nishihama, M., Tewari, K. P., Tilton, J. C., and Isaacman, A. R.: Suomi NPP VIIRS prelaunch and on-orbit geometric calibration and characterization, J. Geophys. Res. Atmos., 118, 11508-11521, doi:10.1002/jgrd.50873, 2013

Xiong, X., Che, N., Barnes, W., Xie, X., Wang, L., and Qu, J.: Status of Aqua MODIS spatial characterization and performance, Proc. SPIE 6361, Sensors, Systems, and Next-Generation Satellites X, 63610T, doi:10.1117/12.687162, 2006. 